\title{
RECURRENT AND SPORADIC FORBUSH DECREASES DURING SOLAR CYCLES 23-24
}

\author{
A.A. Melkumyan \\ Gubkin Russian State University of Oil and Gas \\ (National Research University), \\ Moscow, Russia, amelkumyan6@gmail.com
}

\section{A.V. Belov}

Pushkov Institute of Terrestrial Magnetism, Ionosphere, and Radio Wave Propagation, RAS,

Troitsk, Moscow, Russia, abelov@izmiran.ru

\section{M.A. Abunina}

Pushkov Institute of Terrestrial Magnetism, Ionosphere, and Radio Wave Propagation, RAS,

Troitsk, Moscow, Russia, abunina@izmiran.ru

\section{A.A. Abunin}

Pushkov Institute of Terrestrial Magnetism, Ionosphere, and Radio Wave Propagation, RAS,

Troitsk,Moscow, Russia,abunin@izmiran.ru

\section{E.A. Eroshenko}

Pushkov Institute of Terrestrial Magnetism, Ionosphere, and Radio Wave Propagation, RAS,

Troitsk, Moscow, Russia,erosh@izmiran.ru

\section{V.A. Oleneva}

Pushkov Institute of Terrestrial Magnetism, Ionosphere, and Radio Wave Propagation, RAS,

Troitsk, Moscow,Russia,olene@izmiran.ru

V.G. Yanke

Pushkov Institute of Terrestrial Magnetism, Ionosphere, and Radio Wave Propagation, RAS,

Troitsk, Moscow, Russia,yanke@izmiran.ru

\begin{abstract}
A comparison has been made between recurrent (associated with high-speed streams from coronal holes) and sporadic (caused by interplanetary coronal mass ejections (ICMEs)) Forbush decreases (FDs) in solar cycles 23 and 24 (as well as in the maxima of these cycles and the minimum between them). Forbush Effects and Interplanetary Disturbances database created and maintained in IZMIRAN provided a large number of events (about 1700 isolated FDs, among them 350 recurrent FDs and 207 sporadic FDs selected with high reliability), which allowed us to apply statistical methods. The results revealed that sporadic FDs prevailed in the maxima of the cycles; recurrent FDs, in the minimum between the cycles. FD parameters (magnitude, decrease rate, anisotropy) are larger for sporadic events than for recurrent ones, especially in the maxima of the cycles. FD magnitude is greater in the maxima than in the minimum for sporadic events, and it changes weakly for recurrent ones. The solar wind velocity is on average greater for recurrent events than for sporadic ones; it is larger for recurrent FDs in the minimum and for sporadic FDs in the maxima. The magnetic field is stronger for sporadic FDs than for recurrent ones in the maxima and it is approximately equal for both types of
\end{abstract}

\section{INTRODUCTION}

Forbush decrease (FD) is a change in intensity and anisotropy of cosmic rays (CR) in large-scale solar wind (SW) disturbances. During FDs, CR intensity decreases quite rapidly; the decrease is generally followed by a slower recovery [Dorman, 1963; Iucci et al.,1979; Forbush, 1937; Lockwood, 1971]. Depending on the type of interplanetary medium disturbances, FDs are divided into two groups: sporadic and recurrent. The former are caused by interplanetary coronal mass ejections (ICMEs), associated with coronal mass ejections (CMEs) [Cane, 2000]; the latter, by high-speed streams (HSS) events in the minimum. The magnetic field of ICMEs is weaker in the current solar cycle than in the previous one. The duration of the FD main phase is less in the maxima for both types of events; sporadic FDs developed significantly faster than recurrent ones in the maximum of cycle 23 .

Keywords: Forbush decrease, solar wind, interplanetary magnetic field, coronal mass ejections, coronal holes, solar cycle, statistical analysis. 
2016; Dumbović et al., 2012, 2016]. Abunin et al. [2012] have analyzed hundreds of FDs, divided into two groups: SSC group - events with sudden storm commencement; no-SSC group - events with a gradual onset. It was assumed that the SSC events were largely driven by ICMEs, whereas the no-SSC events were mainly caused by HSS from CHs. Events in these two groups have been shown to be significantly different: the SSC FDs exhibit a much more abrupt and deep reduction in CR intensity than the no-SSC FDs having a symmetrical and smooth temporal profile. Dumbović et al. [2012] have calculated linear regression models and correlation coefficients between FD magnitude, SW velocity and interplanetary magnetic field (IMF) intensity, as well as time characteristics of the events (the time between FD onset and beginning of IMF increase; the time between minimum CR intensity and maximum IMF, etc.). They analyzed different groups of events: related and unrelated to a shock wave; recurrent, driven by coronal interaction regions (CIR), and sporadic, caused by ICMEs; mixed events. The time between FD onset and beginning of IMF increase was shown to be equal for all the groups of events under study; FDs in the CIR group are characterized by a longer time interval between minimum CR intensity and maximum IMF than in the ICME group.

Results of the study of FD number and magnitude in different solar activity phases have also been reported in many papers [Melkumyan et al., 2018b; Belov et al., 2005; Belov, 2009; Lingri at al., 2016; Storini et al., 1997; Thakur, 2015]. Belov [2009] has compared the number of FDs of different magnitudes (greater than $3 \%$ and greater than $5 \%$ ) for solar cycles 19-23. In particular, it was found that large FDs occurred in solar cycle 23 more often than in any other cycles of the period of interest; they often occurred in series, and each series referred to a burst of solar activity. Thakur [2015] has reported results of the study of the statistical relationship between parameters of FDs caused by magnetic clouds (MCs) and SW parameters in solar cycles 23 and 24. It was shown that $31 \%$ of FDs in solar cycle 23 and only $17 \%$ of FDs in solar cycle 24 had a magnitude greater than $3 \% ; 22 \%$ of MCs in cycle 23 and only $10 \%$ of MCs in cycle 24 had a maximum magnetic induction over 20 nT. Thus, MCs in solar cycle 24 have a weaker magnetic field than in solar cycle 23, and, as expected, cause weaker FDs. Lingri et al. [2016] have analyzed sufficiently large FDs (greater than $2 \%$ for $10 \mathrm{GV}$ rigidity) during the deep minimum between cycles 23 and 24, as well as during the ascending phase of solar activity and in the maximum of cycle 24. It was shown that FDs greater than $2 \%$ were associated with fast ICMEs and shock waves, which they generated; the ascending phase of solar cycle 24 featured a sufficiently large number of FDs, but among them there were no major events.

In this paper, we not only compare FDs associated with solar sources of two different types (CH and CME), but also conduct a comparative analysis of recurrent and sporadic FDs in different solar cycles and during different phases of solar activity. The physical meaning of any phenomenon can be found more reliably, the more information, including statistical, we have about this phenome- non. Despite the long-term study of FDs and the large number of articles about them, there is not much reliable statistical information on FDs. The purpose of this study is to gain, store, and check such information. Our task is to compare parameters of Forbush decreases and the largescale SW disturbances which caused the FDs for two groups of events (associated with coronal holes and coronal mass ejections) in different solar activity phases during the last two solar cycles. The considerable amount of initial data allows us to use statistical methods to accomplish the task.

\section{DATA AND METHODS}

We have used FD parameters from the Forbush Effects and Interplanetary Disturbances (FEID) database created and maintained in IZMIRAN [http://spaceweather.izmiran.ru/eng/dbs.html]. The FEID database contains information about FDs from 1957. The FD parameters were obtained from the worldwide neutron monitor network, using the global survey method, for particles with $10 \mathrm{GV}$ rigidity [Belov et al., 2018a; Belov et al., 2018b]. The FEID database also contains information on solar activity and interplanetary medium parameters, as well as on geomagnetic indices. The information about solar flares was gained from GOES satellite measurements [http://www.swpc.noaa.gov/ftpdir/ lists/xray]. The information on SW parameters was taken from the OMNI database [http://omniweb.gsfc.nasa. gov/ow.html].

The comparison between FDs driven by ICMEs and HSS from CHs assumes that each event develops under the influence of a single source. We have tried to identify such events, selecting FDs from the database whose onsets occurred more than $36 \mathrm{hrs}$ before the subsequent event and at least $60 \mathrm{hrs}$ after the previous event (if the previous FD had a magnitude greater than $1.4 \%$ ). To divide the events into two groups (recurrent and sporadic FDs), i.e. to relate FD to a solar source, we conducted a complex analysis of solar and interplanetary data [Belov, 2009 ; Melkumyan et al., 2018a]. We used coronagraph data, images, and video images of the Sun, databases, and catalogues: [Richardson, Cane, 2010; Tlatov et al., 2014; http://www.srl.caltech.edu/ACE/ASC/DATA/ level3/icmetable2.htm;

http://www.solen.info/solar/coronal holes.html; https://cdaw.gsfc.nasa.gov/CME_list]. Since we can make a confident identification only when we have sufficiently complete and reliable data, all the selected recurrent and sporadic events are assigned to a relatively recent period (from early 1997).

In this study, to compare FD properties, we used three groups of events: the FULL group (FDs from only one solar source, 1677 events from May 1996 to December 2015); the $\mathrm{CH}$ group (recurrent FDs associated with $\mathrm{CHs}, 350$ events); the CME group (sporadic FDs associated with CMEs, 207 events). To examine the dependence of FDs on solar activity phase, from each group we selected subgroups of events: SC 23 (solar cycle 23); SC 24 (solar cycle 24); SC 23-24 (solar cycles 23 and 24), Max 23 (maximum of solar cycle 23); Min 23-24 (minimum between solar cycles 23 and 24); 
Max 24 (maximum of solar cycle 24). As parameters describing each event we used maximum values of IMF induction $\left(B_{\max }\right)$, SW velocity $\left(V_{\max }\right), \mathrm{CR}$ intensity variation $\left(A_{\mathrm{F}}-\mathrm{FD}\right.$ magnitude), as well as maximum equatorial component of CR anisotropy $\left(A_{x y \max }\right)$ and maximum hourly decrease in CR intensity variation $\left(\left|D_{\min }\right|-\mathrm{FD}\right.$ decrease rate). We also used parameters of FD development in time, namely, time intervals between FD onset and the time of the maximum value of: CR intensity variation ( $T_{\min }$ - the duration of FD main phase), equatorial component of CR anisotropy $\left(T_{\text {Axymax }}\right)$, and hourly decrease in CR intensity $\left(T_{D \min }\right)$. The considerable amount of data allowed us to employ statistical methods to study the above parameters in different groups and subgroups of events: we constructed histograms, calculated means, medians, $25 \%$ and $75 \%$ interquartile ranges [Corder, Foreman, 2009].

\section{RESULTS AND DISCUSSION}

Table 1 shows the number of FDs, and FD distribution parameters for all events during different phases of solar activity in the last two solar cycles.

Solar cyclicity reveals itself in the number and magnitude of FDs: at high solar activity they are stronger than at low solar activity (in the maxima of cycles 23 and 24 FD distribution medians are $1.7 \%$ and $1.4 \%$; in the minimum between the cycles, $1.1 \%$; the number of FDs is 513, 511, and 425 respectively).

Table 2 presents statistical characteristics of FD and SW parameters in the FULL, CH, and CME groups. For all the parameters except the SW velocity, medians and interquartile ranges are significantly larger in the CME group than in the $\mathrm{CH}$ group. For $A_{x y \max }$ and $\left|D_{\min }\right|$, this difference is especially pronounced: the $25 \%$ quartile in the CME group and the $75 \%$ quartile in the $\mathrm{CH}$ group virtually match.

Thus, the distributions of parameters of recurrent and sporadic events differ significantly. For the SW velocity the median is larger in the $\mathrm{CH}$ group (565 $\mathrm{km} / \mathrm{s})$ than in the CME group $(492 \mathrm{~km} / \mathrm{s})$; the interquartile range is slightly wider in the $\mathrm{CH}$ group (481-643 $\mathrm{km} / \mathrm{s}$ ) than in the CME group (428-582 km/s), but the maximum SW velocity is observed in the CME group $(959 \mathrm{~km} / \mathrm{s})$. Thus, the SW velocity as a whole is higher in the $\mathrm{CH}$ group, but the fastest interplanetary disturbances are caused by CMEs.

Figures 1 and 2 show histograms of FDs in the FULL, CH, and CME groups; Figure 3, FDs for the solar minimum between cycles 23 and 24 and for the maximum of cycle 23. All the distributions are characterized by a positive asymmetry; the distributions for the FULL and $\mathrm{CH}$ groups and solar minimum are peaked; the distribution for the CME group and maximum of solar cycle 23 are flat-topped; the distribution for the FULL and CME groups and for the maximum of cycle 23 are long-tailed (the presence of the "tail" of the distribution in the region of large values indicates sporadic FDs [Melkumyan et al., 2018c]). Since the distributions corresponding to the lognormal law have
Table 1

Statistical parameters of FD distribution in the maximum of solar cycle 23 , minimum between cycles 23 and 24 , and maximum of cycle 24 for events caused by both one and several solar sources

\begin{tabular}{|c|c|c|c|c|c|c|c|}
\hline \multirow{2}{*}{$\begin{array}{l}\text { Period of } \\
\text { solar } \\
\text { activity }\end{array}$} & \multirow[b]{2}{*}{$\begin{array}{l}\overrightarrow{0} \\
\tilde{0} \\
\tilde{0}\end{array}$} & \multirow[b]{2}{*}{$\overrightarrow{\tilde{\mid c}}$} & \multirow[b]{2}{*}{ 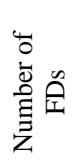 } & \multicolumn{4}{|c|}{ FD magnitude, $\%$} \\
\hline & & & & Mean & 䓌 & Min & $\operatorname{Max}$ \\
\hline Max 23 & 1999 & 2002 & 513 & $2.41 \pm 0.10$ & 1.7 & 0.3 & 16.0 \\
\hline Min 23-24 & 2006 & 2010 & 425 & $1.32 \pm 0.04$ & 1.1 & 0.3 & 12.1 \\
\hline Max 24 & 2012 & 2015 & 511 & $1.85 \pm 0.07$ & 1.4 & 0.4 & 14.8 \\
\hline
\end{tabular}

Table 2

Median, interquartile range boundaries $(25 \%-75 \%)$, minimum and maximum of FD and interplanetary medium parameters in FULL (FDs caused only by one solar source), $\mathrm{CH}$ (recurrent FDs), and CME (sporadic FDs) groups

\begin{tabular}{|c|c|c|c|c|c|}
\hline Parameters & Group & Med & $25 \%-75 \%$ & Min & Max \\
\hline \multirow{3}{*}{$A_{\mathrm{F}}, \%$} & FULL & 1.1 & $0.8-1.7$ & 0.3 & 13.8 \\
\cline { 2 - 6 } & $\mathrm{CH}$ & 1.1 & $0.8-1.5$ & 0.4 & 3.6 \\
\cline { 2 - 6 } & $\mathrm{CME}$ & 2.1 & $1.1-4.0$ & 0.3 & 13.8 \\
\hline \multirow{3}{*}{$\mid D_{\min }, \% / \mathrm{hr}$} & FULL & 0.26 & $0.21-0.35$ & 0.10 & 4.06 \\
\cline { 2 - 6 } & $\mathrm{CH}$ & 0.25 & $0.21-0.30$ & 0.11 & 1.02 \\
\cline { 2 - 6 } & $\mathrm{CME}$ & 0.41 & $0.28-0.64$ & 0.15 & 4.06 \\
\hline \multirow{3}{*}{$A_{x y}$ max,$\%$} & FULL & 1.12 & $0.90-1.43$ & 0.44 & 5.26 \\
\cline { 2 - 6 } & $\mathrm{CH}$ & 0.99 & $0.83-1.18$ & 0.47 & 2.43 \\
\cline { 2 - 6 }$V_{\max , \mathrm{km} / \mathrm{s}}$ & $\mathrm{CME}$ & 1.62 & $1.21-2.09$ & 0.56 & 5.26 \\
\hline & FULL & 487 & $421-586$ & 296 & 959 \\
\cline { 2 - 6 } & $\mathrm{CH}$ & 565 & $481-643$ & 331 & 907 \\
\hline \multirow{3}{*}{$B_{\max , \mathrm{nT}} \mathrm{CME}$} & 492 & $428-582$ & 327 & 959 \\
\cline { 2 - 6 } & FULL & 10.4 & $8.0-13.5$ & 3.7 & 65.6 \\
\cline { 2 - 6 } & $\mathrm{CH}$ & 11.2 & $9.0-13.8$ & 4.3 & 24.9 \\
\cline { 2 - 6 } & $\mathrm{CME}$ & 13.8 & $9.7-20.2$ & 4.7 & 65.6 \\
\hline
\end{tabular}

Note. $A_{\mathrm{F}}$ is the FD magnitude; $\left|D_{\min }\right|$ is the FD decrease rate; $A_{x y \max }$ is the maximum equatorial component of CR anisotropy; $V_{\max }$ is the maximum SW velocity; $B_{\max }$ is the maximum IMF induction.

a positive asymmetry, we tested the hypothesis about lognormal FD distribution, using the KolmogorovSmirnov test, and obtained a negative result.

The comparison of histograms in Figures 2 and 3 allows us to conclude that FDs during solar minimum are generally recurrent, whereas in the maximum of solar cycle 23 they are largely sporadic.

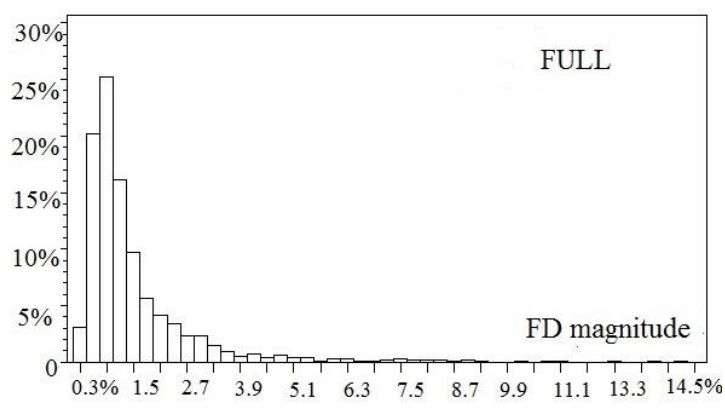

Figure 1. FD distribution, \%, for FULL events, $\%$ 


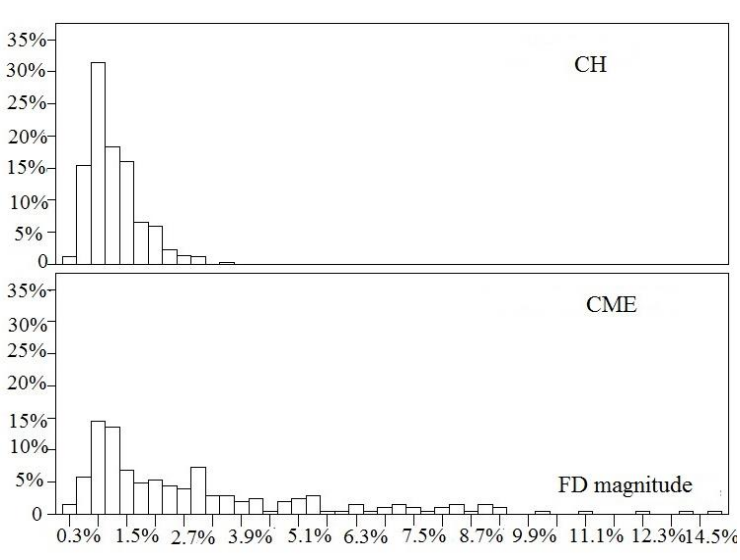

Figure 2. The same as in Figure 1 for events of the $\mathrm{CH}$ (top) and CME (bottom) groups

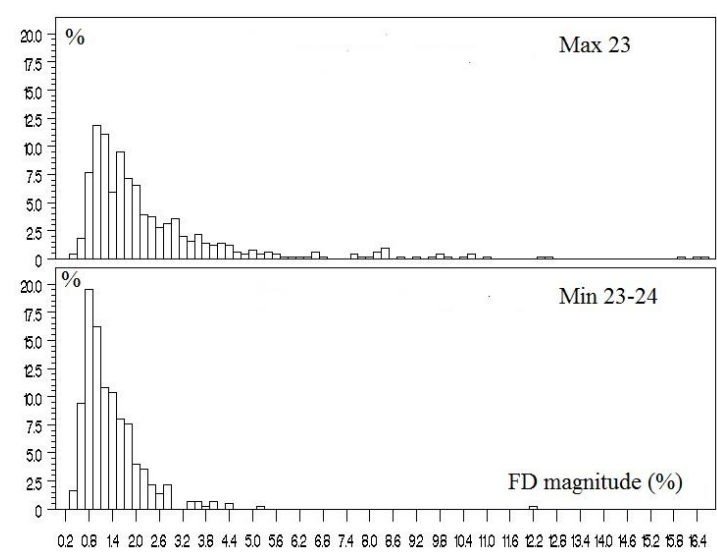

Figure 3. FD distributions for the solar minimum between cycles 23 and 24 and for the maximum of cycle 23

Table 3 and Figures 4-8 show mean values; and Table 4, median, minimum, and maximum FD and SW parameters in $\mathrm{CME}$ and $\mathrm{CH}$ groups for solar cycles 23 and 24. Tables 3, 4, and Figures 4-6 suggest that FD parameters (magnitude, decrease rate, maximum equatorial CR anisotropy) for sporadic events are on average higher than for recurrent ones in all solar activity phases. During the solar minimum, the difference between the mean FD parameters in the two groups is substantially smaller than that during solar maxima (especially for FD magnitudes). The mean FD magnitude in the CME group is practically identical in the maxima of cycles 23 and 24 and significantly smaller in the minimum between the cycles. In the $\mathrm{CH}$ group, the mean FD magnitudes are similar in all solar activity phases, which is likely to be due to the small change in the basic characteristics and geoeffectiveness of low-latitude coronal holes.

The data in Tables 3 and 4 show that the SW velocity is on average higher for cycle 23 than for 24; in all solar activity phases the mean SW velocity is higher for recurrent events than for sporadic ones; the difference between the velocities in the two groups of events is much greater in the solar minimum (medians are 567 and $408 \mathrm{~km} / \mathrm{s}$ respectively).

In this case, for recurrent events the median velocity in the solar minimum is greater than in maxima; and for sporadic events, vice versa. Thus, in the solar minimum

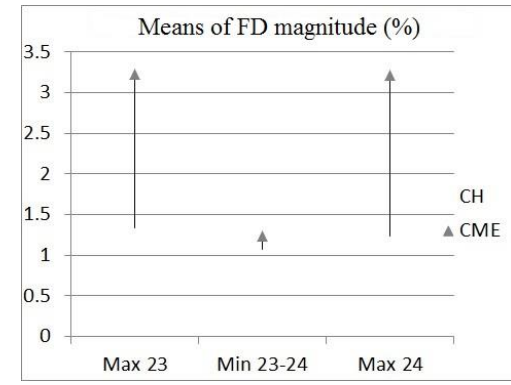

Figure 4. Mean FD magnitudes in the maxima of cycles 23 and 24 and in the minimum between them

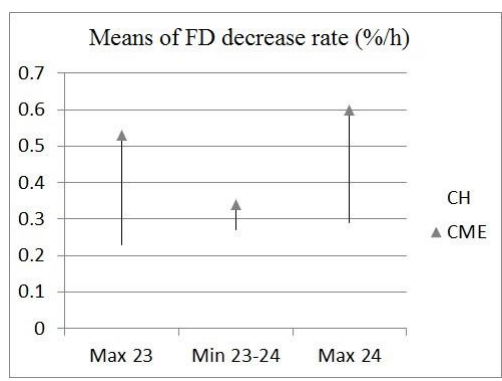

Figure 5. The same as in Figure 4 for the maximum FD decrease rate

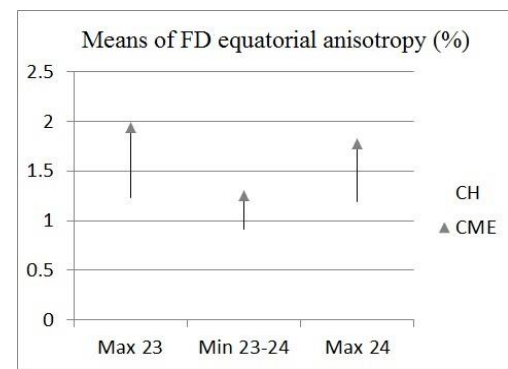

Figure 6. The same for the maximum equatorial anisotropy component

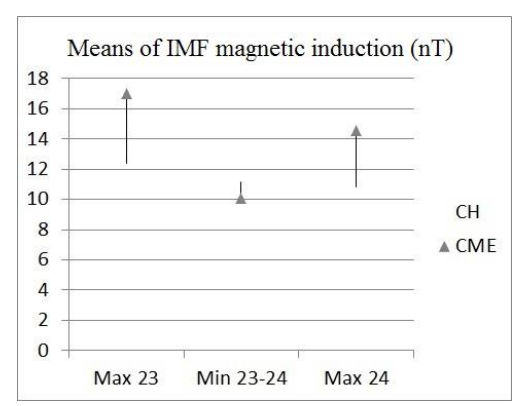

Figure 7. The same for the maximum IMF induction

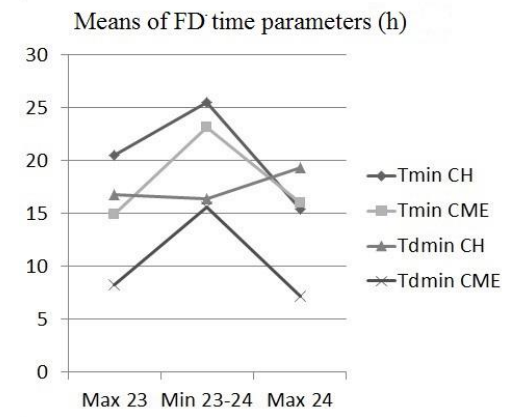

Figure 8. The same for the duration of the FD main phase $\left(T_{\min }\right)$ and the time from the FD onset to the moment of detection of the FD decrease rate $\left(T_{D \min }\right)$ 
The number of events and means of FD and interplanetary medium parameters

\begin{tabular}{|c|c|c|c|c|}
\hline & \multicolumn{2}{|c|}{ SC 23} & \multicolumn{2}{|c|}{ Max 23} \\
\hline & $\mathrm{CH}$ & CME & $\mathrm{CH}$ & CME \\
\hline$N$ & 212 & 136 & 34 & 70 \\
\hline$A_{\mathrm{F}}, \%$ & $1.27 \pm 0.04$ & $3.20 \pm 0.24$ & $1.40 \pm 0.12$ & $3.23 \pm 0.33$ \\
\hline$\left|D_{\min }\right|, \% / \mathrm{hr}$ & $0.27 \pm 0.01$ & $0.60 \pm 0.05$ & $0.24 \pm 0.01$ & $0.53 \pm 0.05$ \\
\hline$A_{x y \max }, \%$ & $1.05 \pm 0.02$ & $1.90 \pm 0.08$ & $1.26 \pm 0.07$ & $1.94 \pm 0.09$ \\
\hline$T_{\min }, \mathrm{hr}$ & $23.1 \pm 1.2$ & $15.6 \pm 1.08$ & $21.9 \pm 2.8$ & $14.9 \pm 1.6$ \\
\hline$T_{D \min }, \mathrm{hr}$ & $17.6 \pm 0.9$ & $9.1 \pm 1.0$ & $17.6 \pm 2.4$ & $8.3 \pm 1.4$ \\
\hline$T_{\text {Axymax }}, \mathrm{hr}$ & $20.0 \pm 1.1$ & $16.3 \pm 1.2$ & $26.8 \pm 3.0$ & $16.0 \pm 1.6$ \\
\hline$V_{\max }, \mathrm{km} / \mathrm{s}$ & $593 \pm 7$ & $539 \pm 11$ & $560 \pm 16$ & $524 \pm 12$ \\
\hline \multirow[t]{3}{*}{$B_{\max }, \mathrm{nT}$} & $12.54 \pm 0.24$ & $17.56 \pm 0.85$ & $12.69 \pm 0.57$ & $17.00 \pm 1.02$ \\
\hline & \multicolumn{2}{|c|}{$\mathrm{SC} 24$} & \multicolumn{2}{|c|}{ Min 23-24 } \\
\hline & $\mathrm{CH}$ & CME & $\mathrm{CH}$ & CME \\
\hline$N$ & 138 & 71 & 165 & 25 \\
\hline$A_{\mathrm{F}}, \%$ & $1.14 \pm 0.04$ & $2.74 \pm 0.29$ & $1.19 \pm 0.04$ & $1.89 \pm 0.40$ \\
\hline$\left|D_{\min }\right|, \% / \mathrm{hr}$ & $0.27 \pm 0.01$ & $0.54 \pm 0.05$ & $0.27 \pm 0.01$ & $0.39 \pm 0.06$ \\
\hline$A_{x y \max }, \%$ & $1.02 \pm 0.02$ & $1.61 \pm 0.07$ & $0.92 \pm 0.02$ & $1.29 \pm 0.15$ \\
\hline$T_{\min }, \mathrm{hr}$ & $21.5 \pm 1.4$ & $18.41 \pm 1.48$ & $25.5 \pm 1.2$ & $23.6 \pm 2.7$ \\
\hline$T_{D \min }, \mathrm{hr}$ & $16.0 \pm 1.3$ & $10.4 \pm 1.4$ & $16.4 \pm 1.1$ & $16.0 \pm 2.6$ \\
\hline$T_{\text {Axymax }}, \mathrm{hr}$ & $16.5 \pm 1.3$ & $13.2 \pm 1.5$ & $19.1 \pm 1.3$ & $20.5 \pm 2.9$ \\
\hline$V_{\max }, \mathrm{km} / \mathrm{s}$ & $529 \pm 9$ & $489 \pm 13$ & $559 \pm 8$ & $459 \pm 26$ \\
\hline \multirow[t]{3}{*}{$B_{\max }, \mathrm{nT}$} & $10.47 \pm 0.27$ & $13.89 \pm 0.92$ & $11.17 \pm 0.26$ & $10.52 \pm 0.75$ \\
\hline & \multicolumn{2}{|c|}{ SC 23-24 } & \multicolumn{2}{|c|}{$\operatorname{Max} 24$} \\
\hline & $\mathrm{CH}$ & CME & $\mathrm{CH}$ & CME \\
\hline$N$ & 350 & 207 & 47 & 39 \\
\hline$A_{\mathrm{F}}, \%$ & $1.22 \pm 0.03$ & $3.04 \pm 0.18$ & $1.26 \pm 0.09$ & $3.31 \pm 0.45$ \\
\hline$\left|D_{\min }\right|, \% / \mathrm{hr}$ & $0.27 \pm 0.01$ & $0.58 \pm 0.04$ & $0.30 \pm 0.02$ & $0.61 \pm 0.07$ \\
\hline$A_{x y \max }, \%$ & $1.04 \pm 0.02$ & $1.80 \pm 0.06$ & $1.21 \pm 0.05$ & $1.83 \pm 0.10$ \\
\hline$T_{\min }, \mathrm{hr}$ & $22.5 \pm 0.9$ & $16.5 \pm 0.9$ & $16.0 \pm 2.5$ & $16.5 \pm 2.0$ \\
\hline$T_{D \min }, \mathrm{hr}$ & $16.9 \pm 0.8$ & $9.5 \pm 0.8$ & $19.1 \pm 2.4$ & $7.6 \pm 1.7$ \\
\hline$T_{\text {Axymax }}, \mathrm{hr}$ & $18.7 \pm 0.9$ & $15.2 \pm 0.9$ & $16.4 \pm 2.4$ & $10.4 \pm 1.8$ \\
\hline$V_{\max }, \mathrm{km} / \mathrm{s}$ & $568 \pm 6$ & $522 \pm 9$ & $549 \pm 16$ & $510 \pm 18$ \\
\hline$B_{\max }, \mathrm{nT}$ & $11.73 \pm 0.19$ & $16.31 \pm 0.65$ & $11.03 \pm 0.43$ & $15.25 \pm 1.22$ \\
\hline
\end{tabular}

Note. $N$ is the number of events; $A_{\mathrm{F}}$ is the FD magnitude; $\left|D_{\min }\right|$ is the FD decrease rate, $A_{x y \max }$ is the maximum equatorial CR anisotropy; $T_{\min }$ is the FD main phase duration; $T_{D \min }$ is the time between FD onset and maximum decrease in CR intensity; $T_{A x y m a x}$ is the time between FD onset and maximum equatorial CR anisotropy; $V_{\max }$ and $B_{\max }$ are the maximum SW velocity and IMF induction.

recurrent FDs are largely caused by high-speed particle fluxes, whereas sporadic FDs are mainly driven by lowspeed ICMEs.

According to Tables 3, 4, and Figure 7, the difference between maximum IMF induction for recurrent and sporadic events in the minimum between cycles 23 and 24 is statistically insignificant. For sporadic events, the median IMF induction in the maxima of cycles $23(14.4 \mathrm{nT})$ and $24(13.1 \mathrm{nT})$ is larger than that in the minimum between the cycles $(9.9 \mathrm{nT})$; the mean induction of the ICME magnetic field in the current cycle $(13.89 \pm 0.92 \mathrm{nT})$ is smaller than that in the previous one $(17.56 \pm 0.85 \mathrm{nT})$. A possible explanation for the weakening of the magnetic field of interplanetary disturbances is given in [Gopalswamy et al., 2015]. The authors carried out a comparative analysis of CME parameters and explained the difference in their geoeffectiveness in solar cycles 23 and 24. The quieter space weather in cycle 24 is due to the anomalous expansion of CMEs, caused by the low pressure of the ambient SW in the inner heliosphere.
Table 3 and Figure 8 present mean time characteristics of FDs for recurrent and sporadic events in different solar activity phases. The Table and Figure indicate that the duration of the FD main phase $\left(T_{\min }\right)$ within each group of events ( $\mathrm{CH}$ and $\mathrm{CME})$ is significantly shorter in the maxima of cycles 23 and 24 than in the minimum between the cycles.

$T_{\min }$ is much shorter for sporadic events than for recurrent ones in the maximum of cycle 23 and are almost equal for the two groups of events in the minimum between the cycles and in the maximum of cycle 24. In [Abunin et al., 2012; Melkumyan et al., 2018a; Belov, 2009], sporadic FDs have been shown to develop faster than recurrent ones. This study helps to specify this statement: sporadic events develop much faster than recurrent ones in the maximum of cycle 23; the FD main phase has a roughly equal duration for sporadic and recurrent events in the minimum between cycles 23 and 24 and in the maximum of cycle 24 . 
The number of events, median (Med), minimum (Min), and maximum (Max) FD and interplanetary medium parameters

\begin{tabular}{|c|c|c|c|c|c|c|c|c|c|c|c|c|}
\hline & \multicolumn{6}{|c|}{ SC 23} & \multicolumn{6}{|c|}{ Max 23} \\
\hline & \multicolumn{3}{|c|}{$\mathrm{CH}$} & \multicolumn{3}{|c|}{ CME } & \multicolumn{3}{|c|}{$\mathrm{CH}$} & \multicolumn{3}{|c|}{ CME } \\
\hline \multirow[t]{2}{*}{$N$} & \multicolumn{3}{|c|}{212} & \multicolumn{3}{|c|}{136} & \multicolumn{3}{|c|}{34} & \multicolumn{3}{|c|}{70} \\
\hline & Med & Min & Max & Med & Min & Max & Med & Min & Max & Med & Min & Max \\
\hline$A_{\mathrm{F}}, \%$ & 1.2 & 0.4 & 3.6 & 2.1 & 0.3 & 13.8 & 1.2 & 0.6 & 3.6 & 2.2 & 0.6 & 13.8 \\
\hline $\begin{array}{l}\left|D_{\min }\right|, \\
\% / \mathrm{hr}\end{array}$ & 0.25 & 0.11 & 0.80 & 0.41 & 0.15 & 4.06 & 0.22 & 0.11 & 0.47 & 0.36 & 0.15 & 2.10 \\
\hline$A_{x y \max }, \%$ & 1.02 & 0.48 & 2.43 & 1.67 & 0.56 & 5.26 & 1.17 & 0.61 & 2.43 & 1.78 & 0.92 & 4.41 \\
\hline$V_{\max }, \mathrm{km} / \mathrm{s}$ & 605 & 354 & 874 & 511 & 327 & 959 & 543 & 419 & 769 & 512 & 327 & 774 \\
\hline \multirow{3}{*}{$B_{\max }, \mathrm{nT}$} & 12.0 & 6.1 & 24.9 & 14.9 & 4.9 & 65.6 & 12.0 & 6.9 & 21.1 & 14.4 & 7.5 & 65.6 \\
\hline & \multicolumn{6}{|c|}{ SC 24} & \multicolumn{6}{|c|}{ Min 23-24 } \\
\hline & \multicolumn{3}{|c|}{$\mathrm{CH}$} & \multicolumn{3}{|c|}{ CME } & \multicolumn{3}{|c|}{$\mathrm{CH}$} & \multicolumn{3}{|c|}{ CME } \\
\hline \multirow[t]{2}{*}{$N$} & \multicolumn{3}{|c|}{138} & \multicolumn{3}{|c|}{71} & \multicolumn{3}{|c|}{165} & \multicolumn{3}{|c|}{25} \\
\hline & Med & Min & Max & Med & Min & Max & Med & Min & Max & Med & Min & Max \\
\hline$A_{\mathrm{F}}, \%$ & 1.0 & 0.5 & 3.0 & 2.0 & 0.3 & 13.1 & 1.1 & 0.5 & 2.9 & 1.3 & 0.3 & 10.2 \\
\hline $\begin{array}{l}\left|D_{\min }\right| \\
\% / \mathrm{hr}\end{array}$ & 0.24 & 0.14 & 1.02 & 0.38 & 0.15 & 2.35 & 0.25 & 0.13 & 0.80 & 0.31 & 0.15 & 1.56 \\
\hline$A_{x y \max }, \%$ & 0.97 & 0.47 & 2.38 & 1.58 & 0.66 & 3.27 & 0.92 & 0.47 & 1.61 & 1.06 & 0.56 & 4.35 \\
\hline$V_{\max }, \mathrm{km} / \mathrm{s}$ & 515 & 331 & 907 & 453 & 336 & 737 & 567 & 331 & 777 & 408 & 342 & 896 \\
\hline \multirow[t]{3}{*}{$B_{\max }, \mathrm{nT}$} & 9.7 & 4.3 & 21.0 & 11.3 & 4.7 & 40.1 & 10.5 & 4.3 & 21.7 & 9.9 & 4.9 & 19.2 \\
\hline & \multicolumn{6}{|c|}{ SC 23-24 } & \multicolumn{6}{|c|}{ Max 24} \\
\hline & \multicolumn{3}{|c|}{$\mathrm{CH}$} & \multicolumn{3}{|c|}{ CME } & \multicolumn{3}{|c|}{$\mathrm{CH}$} & & $\mathrm{CME}$ & \\
\hline$N$ & & 350 & & & 207 & & & 47 & & & 39 & \\
\hline & Med & Min & Max & Med & Min & Max & Med & Min & $\operatorname{Max}$ & Med & Min & $\operatorname{Max}$ \\
\hline$A_{\mathrm{F}}, \%$ & 1.1 & 0.4 & 3.6 & 2.1 & 0.3 & 13.8 & 1.1 & 0.5 & 3.0 & 2.8 & 0.5 & 13.1 \\
\hline $\begin{array}{l}\left|D_{\min }\right|, \\
\% / \mathrm{hr}\end{array}$ & 0.25 & 0.11 & 1.02 & 0.41 & 0.15 & 4.06 & 0.28 & 0.17 & 1.02 & 0.49 & 0.20 & 2.35 \\
\hline$A_{x y \max }, \%$ & 0.99 & 0.47 & 2.43 & 1.62 & 0.56 & 5.26 & 1.17 & 0.54 & 2.38 & 1.78 & 0.83 & 3.27 \\
\hline$V_{\max }, \mathrm{km} / \mathrm{s}$ & 565 & 331 & 907 & 492 & 327 & 959 & 542 & 369 & 907 & 483 & 336 & 737 \\
\hline$B_{\max }, \mathrm{nT}$ & 11.2 & 4.3 & 24.9 & 13.8 & 4.7 & 65.6 & 10.8 & 5.3 & 19.6 & 13.1 & 5.8 & 40.1 \\
\hline
\end{tabular}

Note. $N$ is the number of events; $A_{\mathrm{F}}$ is the FD magnitude; $\left|D_{\min }\right|$ is the FD decrease rate; $A_{x y \max }$ is the maximum equatorial CR anisotropy; $V_{\max }$ is the maximum SW velocity; $B_{\max }$ is the maximum IMF induction.

\section{CONCLUSIONS}

The use of statistical methods to compare Forbush decreases from solar sources of two types ( $\mathrm{CH}$ and $\mathrm{CME})$ in different solar activity phases in the last two solar cycles led to the following conclusions:

1. In FDs, solar cyclicity manifests itself: at high solar activity, the FD distribution median is significantly higher than that at low solar activity.

2. Distributions of FD and SW parameters differ markedly for events caused by ICMEs (sporadic FDs) and HSS from coronal holes (recurrent FDs). For recurrent events, the mean FD magnitude depends weakly on solar activity phase. It is likely to mean that the main characteristics and geoeffectiveness of low-latitude coronal holes vary only slightly in the solar cycles.

3 . FDs in the solar minimum between solar cycles 23 and 24 are largely caused by high-speed SW streams from coronal holes; and in the maximum of solar cycle 23 , by CME-driven interplanetary disturbances.

4. In solar cycles 23 and 24 in all solar activity phases, FD parameters (FD magnitude, FD decrease rate and maximum equatorial CR anisotropy) are significantly greater for sporadic FDs than for recurrent ones. In the solar maximum, the difference between the parameters of these two groups is substantially larger than in the minimum.

5. The IMF induction is on average much greater for sporadic FDs than for recurrent ones in the maxima of so- lar cycles 23 and 24, and is virtually the same for events of the two types in the minimum between the cycles. The IMF induction for sporadic events is much smaller in cycle 24 than in cycle 23, i.e. magnetic fields of ICMEs in the current cycle is weaker than in the previous one, which is likely to be due to the anomalous expansion of CMEs, caused by the low pressure of ambient SW in the inner heliosphere.

6 . The SW velocity in all solar activity phases is on average significantly higher for recurrent events than for sporadic ones, although the maximum velocities are in the group of events associated with ICMEs. The SW velocity is significantly higher for sporadic events in the maxima of cycles 23 and 24 and slightly higher for recurrent events in the minimum between the cycles. Apparently, in the solar minimum recurrent FDs are largely caused by high-speed SW streams and sporadic FDs are mainly triggered by lowspeed ICMEs. The SW velocity in both recurrent and sporadic events is lower in the current cycle than in the previous one.

7. The analysis of the mean duration of the FD main phase has shown that: a) in the maximum of cycle 23 , sporadic events develop much faster than recurrent ones; b) in the maximum of cycle 24 and in the minimum between the cycles, the development of recurrent and sporadic events has virtually the same duration; c) the duration of the FD main phase in each group of events is longer in the mini- 
mum between cycles 23 and 24 than in the maxima of these cycles.

We are grateful to the teams of the worldwide network of cosmic ray stations providing data from continuous recording of the neutron component [http://cr0.izmiran.ru/ThankYou] as well as from the NMDB database [http://www.nmdb.eu], established under the EU FP7 program (contract No. 213007).

\section{REFERENCES}

Abunin A.A., Abunina M.A., Belov A.V., Eroshenko E.A. Oleneva V.A., Yanke V.G. Forbush effects with a sudden and gradual onset. Geomagnetism and Aeronomy. 2012, vol. 52, no. 3, pp. 292-299. DOI: 10.1134/S0016793212039924.

Badruddin K.A. Study of the cosmic-ray modulation during the passage of ICMEs and CIRs. Solar Phys. 2016, vol. 291, no. 2, pp. 559-580. DOI: 10.1007/s11207-015-0843-4.

Belov A.V. Forbush effects and their connection with solar, interplanetary and geomagnetic phenomena. Proc. IAU Symposium. 2009, no. 257, pp. 119-130.

Belov A.V., Buetikofer R., Eroshenko E.A., Flueckiger E.O., Gushchina R.T., Oleneva V.A., Yanke V.G. Frequency of Forbush effects as an index of solar activity. Proc. $29^{\text {th }}$ ICRC. 2005. V. 1, P. 375-378.

Belov A., Abunin A., Abunina M., Eroshenko E., Oleneva V., Yanke V., Papaioannou A., Mavromichalaki H., Gopalswamy N., Yashiro S. Coronal mass ejections and non-recurrent Forbush decreases. Solar Phys. 2014, vol. 289, no. 10, pp. 3949-3960. DOI: 10.1007/s11207-014-0534-6.

Belov A.V., Eroshenko E.A., Yanke V.G., Oleneva V.A., Abunina M.A., Abunin A.A. Global Survey Method for the world network of neutron monitors. Geomagnetism and Aeronomy. 2018a, vol. 58, no. 3. pp. 356-372. DOI: $10.1134 /$ S0016793218030039.

Belov A.V., Eroshenko E.A., Yanke V.G., Oleneva V.A., Abunina M.A., Abunin A.A., Papaioannou A., Mavromichalaki H. The Global Survey Method applied to ground-level cosmic ray measurements. Solar Phys. 2018b, vol. 293, no. 68. DOI: 10.1007/s11207-018-1277-6.

Bhaskar A., Subramanian P., Vichare G. Relative contribution of the magnetic field barrier and solar wind speed in ICME-associated Forbush decreases. Astrophys. J. 2016, vol. 828, no. 2 , article id. 104,8 p. DOI: $10.3847 / 0004-637 X / 828 / 2 / 104$.

Cane H.V. CMEs and Forbush decreases. Space Sci. Rev. 2000, vol. 93, no. 1-2, pp. 55-77.

Chertok I.M., Abunin A.A., Belov A.V., Grechnev V.V. Dependence of Forbush-decrease characteristics on parameters of solar eruptions. J. Phys. Conf. Ser. 2013, vol. 409, no. 1, article id. 012150. DOI: 10.1088/1742-6596/409/1/012150.

Corder G.W., Foreman D.I. Nonparametric Statistics for Non-Statisticians. New Jersey, John Willey \& Sons, 2009, 264 p.

Dorman L.I. Variatsii kosmicheskikh luchei $i$ issledovanie kosmosa [Cosmic Ray Variation and Space Research]. Moscow, 1963. 1027 p. (In Russian). English edition: Dorman L.I. Cosmic Rays: Variations and Space Explorations. Amsterdam, NorthHolland; New York, American Elsevier, 1974, 675 p.

Dumbović M., Vršnak B., Čalogović J., Župan R. Cosmic ray modulation by different types of solar wind disturbances. Astron. Astrophys. 2012, vol. 538, A28. DOI: 10.1051/00046361/201117710.

Dumbović M., Vršnak B., Čalogović J. Forbush decrease prediction based on remote solar observations. Solar Phys. 2016, vol. 291, no. 1, pp. 285-302. DOI: 10.1134/s11207-015-0819-4.

Forbush S.E. On the effects in the cosmic-ray intensity observed during magnetic storms. Phys. Rev. 1937, vol. 51, pp. $1108-1109$.

Gopalswamy N. Coronal mass ejections: a summary of recent results. Proc. $20^{\text {th }}$ National Solar Physics Meeting, Papradno, Slovakia. 2010, pp. 108-130.
Gopalswamy N., Akiyama S., Yashiro S., Xie H., Mäkelä P., Michalek G. The mild space weather in solar cycle 24. ArXiv. URL: https://arxiv.org/abs/1508.01603 (accessed November 9, 2018).

Iucci N., Parisi M., Storini M., Villoresi G. Forbush decreases: origin and development in the interplanetary space. Nuovo Cimento C. 1979, vol. 2C, pp. 1-52. DOI: 10.1007/BF02507712.

Kryakunova O., Tsepakina I., Nikolaevskiy N., Malimbaev A., Belov A., Abunin A., Abunina M., Eroshenko E., Oleneva V., Yan$\mathrm{ke} \mathrm{V}$. Influence of high-speed streams from coronal holes on cosmic ray intensity in 2007. J. Phys. Conf. Ser. 2013, vol. 409, no. 1, article id. 012181. DOI: 10.1088/1742-6596/409/1/012181.

Lingri D., Mavromichalaki H., Belov A., Eroshenko E., Yanke V., Abunin A., Abunina M. Solar activity parameters and associated Forbush decreases during the minimum between cycles 23 and 24 and the ascending phase of cycle 24. Solar Phys. 2016, vol. 291, no. 3, pp. 1025-1041. DOI: 10.1007/s11207-016-0863-8.

Lockwood J.A. Forbush decreases in the cosmic radiation. Space Sci. Rev. 1971, vol. 12, no. 5, pp. 658-715. DOI: $10.1007 / \mathrm{BF} 00173346$.

Melkumyan A.A., Belov A.V., Abunina M.A., Abunin A.A., Eroshenko E.A., Oleneva V.A., Yanke V.G. Main properties of Forbush effects related to high-speed streams from coronal holes. Geomagnetism and Aeronomy. 2018a, vol. 58, no. 2, pp. 154168. DOI: $10.1134 / \mathrm{s} 0016793218020159$.

Melkumyan A.A., Belov A.V., Abunina M.A., Abunin A.A., Eroshenko E.A., Oleneva V.A., Yanke V.G. Long term changes in the number and magnitude of Forbush effects. Geomagnetism and Aeronomy. 2018b, vol. 58, no. 5, pp. 615-624. DOI: $10.1134 / \mathrm{s} 0016793218050109$.

Melkumyan A.A., Belov A.V., Abunina M.A., Abunin A.A., Eroshenko E.A., Oleneva V.A., Yanke V.G. Size distribution of Forbush effects. Geomagnetism and Aeronomy. 2018c, vol. 58, no. 6, pp. 809-816. DOI: 10.1134/s0016793218050109.

Richardson I.G. Energetic particles and corotating interaction regions in the solar wind. Space Sci. Rev. 2004, vol. 111, no. 3, pp. 267-376. DOI: 10.1023/B:SPAC.0000032689.62830.3e.

Richardson I., Cane H. Near-Earth interplanetary coronal mass ejections during solar cycle 23 (1996-2009): Catalog and summary of properties. Solar Phys. 2010, vol. 264, no. 1, pp. 189-237. DOI: 10.1007/s11207-010-9568-6.

Storini M., Massetti S., Antalova A. To forecast huge Forbush decreases during solar activity cycles. Proc. $25^{\text {th }}$ ICRC. 1997, vol. 1, pp. 409-412.

Thakur N. Smaller Forbush decreases in solar cycle 24: effect of the weak CME field strength? American Geophysical Union, Fall Meeting 2015. id. SH23A-2428.

Tlatov A., Vasil'eva V., Tavastsherna K. Coronal holes in solar cycles 21 to 23. Solar Phys. 2014, vol. 289, no. 4, pp. 13491358. DOI: $10.1007 / \mathrm{s} 11207-013-0387-4$.

URL: http://spaceweather.izmiran.ru/eng/dbs.html (accessed November 9, 2018).

URL: http://www.swpc.noaa.gov/ftpdir/lists/xray (accessed November 9, 2018).

URL: http://omniweb.gsfc.nasa.gov/ow.html (accessed November 9,2018 )

URL: http://www.srl.caltech.edu/ACE/ASC/DATA/level3/ icmetable2.htm (accessed November 9, 2018).

URL: http://www.solen.info/solar/coronal_holes.html (accessed November 9, 2018).

URL: https://cdaw.gsfc.nasa.gov/CME_list (accessed November 9, 2018).

URL: http://cr0.izmiran.ru/ThankYou (accessed November 9, 2018).

URL: http://www.nmdb.eu (accessed November 9, 2018).

How to cite this article

Melkumyan A.A., Belov A.V., Abunina M.A., Abunin A.A., Eroshenko E.A. Oleneva V.A., Yanke V.G. Recurrent and sporadic Forbush decreases during solar cycles 23 and 24. Solar-Terrestrial Physics. 2019. Vol. 5. Iss. 1. P. 28-34. DOI: 10.12737/stp-51201904. 